\title{
Anterior approach to the subaxial cervical spine in children: a brief review
}

\author{
Eli M. Baron, M.D., Christopher M. Loftus, M.D., Alexander R. VacCaro, M.D., \\ ANd Devanand A. DominiQue, M.D.
}

Department of Neurosurgery, Temple University School of Medicine; and Rothman Institute, Department of Orthopedics, Thomas Jefferson Hospital, Philadelphia, Pennsylvania

\begin{abstract}
$\checkmark$ Although it was originally developed to address degenerative problems, including disc herniations and cervical spondylotic myelopathy in the adult population, the anterior approach to the subaxial spine has proven to be useful for select indications in the pediatric population. The authors review indications for surgery, bone grafting, and instrumentation as they pertain to children.
\end{abstract}

KEY WORDS - anterior cervical approach - bone graft • children

$\mathrm{T}$

HE anterior approach to the cervical spine, the technique originally developed and described by Smith and Robinson, ${ }^{27}$ Cloward, ${ }^{10}$ and Bailey and Badgley ${ }^{3}$ in the late 1950s and early 1960s for treatment of cervical disc herniations and spondylotic myelopathy in the adult population, has proven to be quite useful for select indications in children. This approach has been used for correction of congenital, traumatic, and iatrogenic deformity of the cervical spine; to treat cervical disc herniations; and for the surgical management of tumors. Similar surgical strategies in terms of the approach and surgical decision making apply to the pediatric as well as to the adult population. Differences, however, arise when one takes into consideration the fact that the pediatric cervical spine continues to grow from childhood into adolescence, and that standard spinal instrumentation may have too large a profile for general pediatric use. We review the indications for an anterior cervical approach in the pediatric spine and discuss bone grafting, instrumentation, and bioabsorbable technology in relation to the pediatric population.

\section{Indications for Surgery}

\section{Spinal Deformity}

The anterior cervical approach has been used in the pediatric population for the treatment of deformity caused by a wide variety of disorders, including congenital anom-

Abbreviations used in this paper: $\mathrm{ACDF}=$ anterior cervical discectomy and fusion; AP = anteroposterior; BMP = bone morphogenetic protein; $\mathrm{CT}=$ computerized tomography; $\mathrm{PEEK}=$ polyetheretherketone; $\mathrm{VB}=$ vertebral body. alies of the spine, trauma, connective tissue disorders, and iatrogenic disorders. In 1978, Holmes and Hall ${ }^{16}$ first described using the anterior approach to the cervical spine in the pediatric population. They reported on an 8-month-old boy who had numerous congenital anomalies of the cervical spine. The child underwent a $360^{\circ}$ fusion that was completed in two stages. He underwent anterior fusion 1 month before a posterior fusion was performed. The anterior approach was used for multilevel vertebrectomies, and $\mathrm{C} 1-5$ fusion was completed using autologous tibial strut graft.

In a dissimilar report, Deburge and Briard ${ }^{11}$ described a 14-year-old girl with a C-7 hemivertebra that resulted in coronal plane deformity in which her head was inclined $30^{\circ}$ to the right. The patient underwent staged procedures, including a partial excision of the C-7 vertebra to create a $30^{\circ}$ wedge, posterior excision of components of her hemivertebra, and manual reduction of the surgically created gap, followed by plate stabilization of the adjacent VBs via an anterior approach to maintain the deformity correction.

The anterior cervical approach has been used to treat iatrogenic kyphotic deformity. In their paper discussing theories of cervical fusion for instability in the pediatric population, Holmes and Hall ${ }^{16}$ described a 4-year-old boy who presented with subaxial neck pain after undergoing a C1-4 laminectomy. Subluxations at C2-3 and C3-4 were diagnosed, and he subsequently underwent anterior cervical fusion from C-2 to C-5 using autogenous bone graft and halo vest immobilization, with good results at 2.5 years.

The anterior cervical approach has also been used in the pediatric population for kyphotic deformity resulting from 
connective tissue disorders. Piccirilli and Chadduck ${ }^{24}$ described the case of a child with Morquio syndrome and subsequent severe kyphotic deformity at C3-5. After being placed in halo traction, the child underwent $\mathrm{C} 3-5$ vertebrectomy and removal of large amounts of fibrous tissue and the posterior longitudinal ligament. A full-thickness calvarial bone graft was then used, joined by cables to function as a strut.

Larson syndrome, a rare connective tissue disorder, also may present with cervical kyphosis requiring surgical intervention. ${ }^{4,18}$ Banks, et al., ${ }^{4}$ described the case of a 13 -year-old boy with Larsen syndrome and consequent cervical kyphotic myelopathy. This patient underwent anterior-posterior fusion, with occipitocervical thoracic fusion performed first with laminectomy, followed by a C5-7 corpectomy with autogenous iliac crest bone graft and anterior cervical plate placement.

\section{Spinal Trauma}

The anterior cervical approach to the spine has perhaps been used most widely in the treatment of pediatric spinal trauma (Fig. 1). Approximately $72 \%$ of cervical spine injuries occur in children younger than 8 years of age, with the vast majority of the injuries occurring between the occiput and C-3. By the age of 10 years, most children's cervical spines are more similar to those of adults in terms of proportion, and in children older than 10 to 12 years of age, the clinical sequelae of trauma are remarkably similar to those found in adults. ${ }^{20}$ Additionally, cervical spine injuries are less common in children as a result of increased elasticity of the child's spinal elements when compared with adults. $^{26}$

The majority of pediatric cervical trauma is treated nonoperatively. Nevertheless, when surgery is recommended, it is usually performed via a posterior approach, because several authors have noted that damage to both superior and inferior cervical VB endplates might result in postoperative instability and subsequent angular deformity. ${ }^{26,29}$ Regardless, there have been several case series in which good to excellent outcomes have been reported for anterior cervical discectomy/corpectomy and fusion.

Wickboldt and Sörensen ${ }^{36}$ reviewed their series of five patients, ages 11 to 14 years, who underwent ACDF. The authors used this procedure to treat traumatic subluxation and instability, with good results. Notably, before surgery, four of these five patients had been immobilized for relatively long periods. The fifth patient, however, was treated with early surgery. Intraoperatively, all five patients were found to have cervical disc ruptures. In these authors' extensive discussion, they noted that the most likely reason that these spines failed to fuse with the aid of halo immobilization was the apparently poor healing capacity of the nuchal ligaments, joint capsules, and intervertebral disc. They also noted that at approximately the age of 8 years, involution of the vascularization of the intervertebral disc begins, further reducing healing capacity. They concluded that early intervention with the anterior cervical approach may be a more successful way to treat dislocation and fracture of the cervical spine.

Shacked, et al. ${ }^{26}$ published a series that included six children, ages 3 to 14 years, in whom the anterior approach was used for decompression and stabilization of traumatic cer- vical injury. Three indications for surgery were cited; they included severe hyperflexion injury with crushed bones of the VB, fracture/dislocation with involvement of the posterior elements and disc, and major anatomical deformity of the cervical spine with cord compression. Additionally, one patient underwent surgical fusion 8 weeks postinjury after halo vest immobilization failed to produce fusion. These authors noted in their results that no postoperative deformity or instability was seen during follow-up visits, although a slight kyphosis was seen in some patients. Additionally, solid fusion was noted on all radiographs obtained at longterm follow-up review. These investigators emphasized the role the shape and fit of the tricortical bone graft plays in achieving optimal radiographic results.

Similarly, Hoffmann, et al., ${ }^{15}$ reported the case of a 6year-old girl who sustained a C3-5 fracture dislocation in addition to unilateral locked facet at $\mathrm{C} 3-4$ following a motor vehicle accident. After closed reduction failed, she underwent an anterior-posterior procedure in which a C-4 vertebrectomy was performed, with subsequent placement of iliac crest bone graft and placement of an AO three-hole tubular titanium plate, because her VBs were deemed too small for standard adult instrumentation. Additionally, the patient underwent posterior spinal fusion. Solid fusion was documented radiographically at 6 months, and her implants were subsequently removed. Good radiological and clinical results were noted at 2.5 years.

Brockmeyer, et al., ${ }^{6}$ reviewed their experience with instrumentation in the pediatric cervical spine. Eleven adolescent patients underwent the anterior cervical approach with plate placement for fractures or ligamentous instability. Iliac crest autograft was used in seven patients, whereas allograft was used in four. Although the majority of the patients did very well, a single instance of repeated operation was noted in a patient who had received allograft for graft settling.

Recently, Dickerman, et al., ${ }^{13}$ reported on an 18-monthold girl who became quadriparetic because of a fall; she was found to have a $C 2-3$ disruption. Subsequently, the patient underwent corpectomy at C-3 and at the base of C-2; autologous rib graft and Macropore (PLA; Macropore, San Diego, CA) were used as the anterior plating system. This was followed by a posterior occiput-C3 fusion with titanium wiring and autologous rib graft. Postoperatively the patient was kept in a halo vest for 4 months, with fusion and neurological recovery resulting.

\section{Disc Herniations}

Given the superior vascularity of the juvenile cervical disc compared with adult discs, and the greater elasticity of the surrounding longitudinal ligaments, disc herniations are a rarity in the pediatric population. Nevertheless, when they do occur, because of the rich microvasculature spontaneous resorption is usually the natural course. ${ }^{19,22}$ On occasion, severe symptoms of radiculopathy or myelopathy may necessitate surgical intervention.

Schaser, et al. ${ }^{25}$ described the case of a 12-year-old child who experienced myelopathy caused by calcified disc herniation at C3-4. The child then underwent C3-4 ACDF in which iliac crest bone grafting was used. At 1-year followup review, the patient was documented to be doing well.

Klippel-Feil syndrome may predispose children to the 

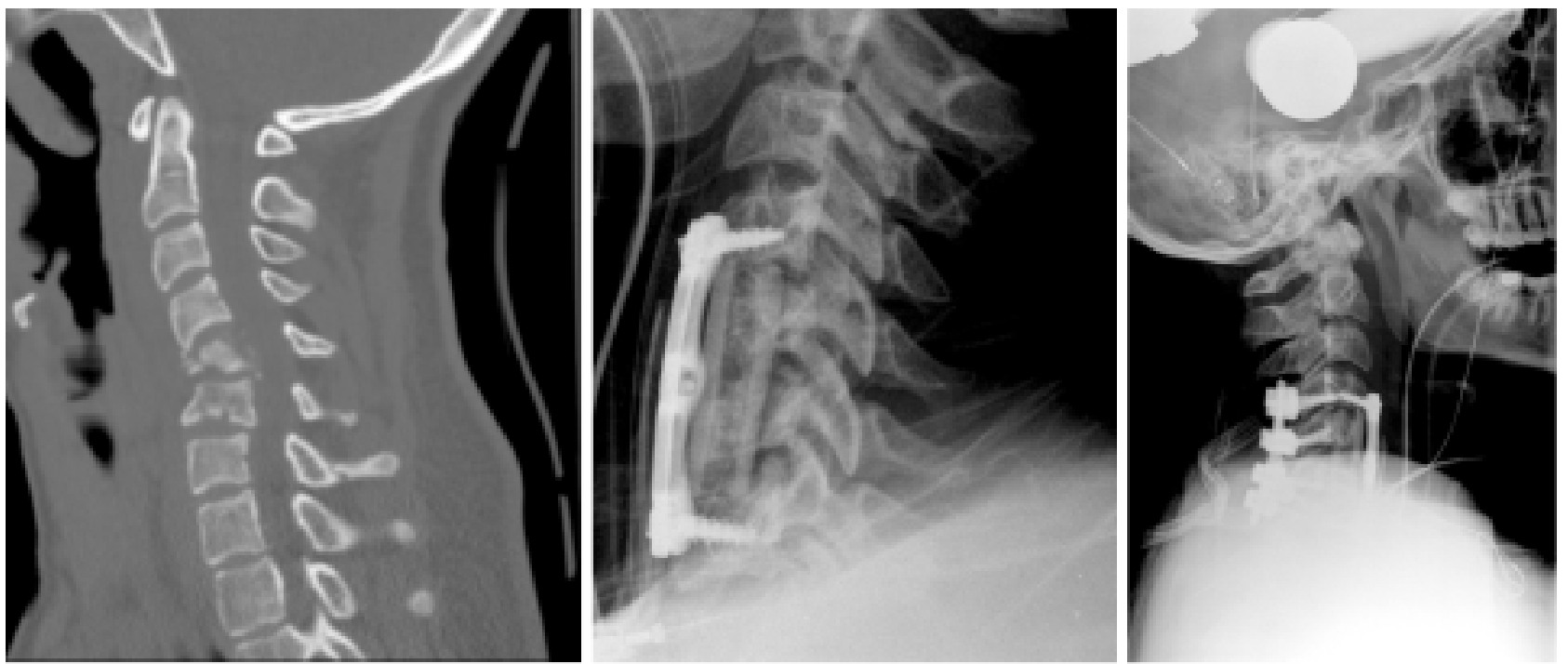

FIG. 1. Left: Sagittal CT reconstruction demonstrating an advanced-stage C5-6 flexion-compression injury in a 6year-old girl after she was involved in a motor vehicle accident. This patient's neurological score was noted to be a Grade A at C-6 on the American Spinal Injury Association scale. Note also the associated spinous process fractures. Center and Right: Lateral plain x-ray films obtained postoperatively. A C5-6 corpectomy was performed using a fibular allograft strut graft filled with corpectomized bone and an anterior cervical plate from C-4 to C-7 (center). A subsequent posterior stabilization procedure was completed using lateral mass screws, C-7 pedicle screws, and rods, with local corpectomized bone as a fusion graft from the anterior procedure (right).

development of cervical disc herniations. Allsopp, et al., ${ }^{1}$ described the case of a child who had experienced cervical myelopathy for 1 year secondary to C3-4 disc herniation, with subsequent spinal cord compression. The child underwent C3-4 ACDF with autograft fusion, and had excellent results at the 1-year follow-up visit.

\section{Tumors of the Spine}

The anterior cervical approach with and without instrumentation has been used for the treatment of pediatric neoplastic disease. In 1979, Fielding, et al.. ${ }^{14}$ conducted a survey of 38 spine surgeons to review their practice regarding anterior cervical vertebrectomy for benign and malignant tumors. Among the 20 patients treated surgically with an anterior approach by this group of surgeons were two children and three adolescents. Pathological entities included benign diseases such as osteoblastoma and malignancies such as neurofibrosarcoma. Autologous bone grafting, including iliac crest, fibula, and tibia, was used in all patients, and all of them underwent combined anterior-posterior fusion, with the exception of a 13-year-old boy who underwent $\mathrm{C}^{-7}-7$ vertebrectomy and fusion with a fibular strut graft. The duration of follow up was 1 to 8 years, and the treatments yielded good to excellent results.

Sohn, et al., ${ }^{28}$ described a 7 -year-old girl who had a vertebra plana at C-4 that was caused by an eosinophilic granuloma. She subsequently underwent C-4 vertebrectomy with autologous iliac crest used as a tricortical strut graft. In addition, a 2-mm-thick miniplate was used for more support, because standard anterior cervical plates were too large for the patient. Postoperatively the patient did well after undergoing halo vest therapy for 3 months. Follow-up review at 2 years showed no problems regarding deformity, and there was also maintenance of cervical lordosis.

\section{Materials and Techniques}

\section{Bone Grafting}

These series from the literature demonstrate considerable use of autogenous bone for anterior cervical fusion in the pediatric population. This has been done primarily with iliac crest, but also with fibula and tibia and rib grafts. Considerable donor site morbidity related to iliac crest bone grafting has been described, including persistent graft site discomfort (as high as 36\% of patients), injury to the lateral femoral cutaneous nerve, painful hematoma, wound infections, and avulsion of the anterior superior iliac spine. ${ }^{12}$ In young children the iliac crest may be quite cartilaginous ${ }^{7}$ and therefore would be an inappropriate source for autograft. Rib grafting is also associated with pain, rare pneumothoraces, and a relative lack of rigidity/strength of the graft. ${ }^{8}$ Thus, practitioners have used calvarial bone grafts as an alternative to more traditional sources, with the advantages being an abundant supply of graft, a decreased rate of resorption after graft placement (seen in animal studies), and reduced postoperative morbidity. ${ }^{7,8}$ For longer constructs, autologous fibula can be used. This also can cause significant local complications, including decrease in sensation in the first dorsal web space of the foot, motor deficits, local site pain, and postoperative tibial stress fractures. ${ }^{21}$

Regardless of the source, autologous bone, in general, has the advantage of being osteogenic, osteoinductive, and osteoconductive. Autograft is more likely to be completely and more quickly incorporated than allograft, because it does not elicit the same immune response that allograft does. ${ }^{21}$ Although pure cancellous bone may have a higher fusion rate than cortical bone, cancellous bone carries only $60 \%$ of the compressive strength of tricortical graft. Thus, cancellous bone grafts alone cannot withstand the compressive forces needed in anterior spinal column stabilization. ${ }^{32}$ 
Even though it is a less attractive alternative in the pediatric population, allograft remains an option. Currently, fresh, frozen, and freeze-dried preparations are most commonly used for adults. Although frozen graft can preserve BMP, viruses present in these grafts are not completely eradicated. Given the fact that no screening method is entirely foolproof, the virus issue may come into consideration with regard to the pediatric population. Fresh-frozen processing decreases compression stress by 10 to $20 \%$, but does not affect the bending or torsional strength of the graft. In freeze-dried preparations, on the other hand, bending strength is decreased by more than 55\% and torsional strength by $39 \%$. Also, these grafts may be prone to longitudinal cracking. Although freeze-dried preparations are theoretically virus free (because the entire viral DNA has been destroyed by the processing), denaturation of BMPs also occurs. In theory, freeze-dried preparations result in potentially decreased fusion rates. ${ }^{21}$

Brockmeyer, et al., ${ }^{6}$ used banked bone in four adolescent patients undergoing the anterior cervical approach and fusion with plate placement for traumatic indications. Notably, in one case repeated operation was necessary because of allograft settling. No problems were seen in their seven patients who underwent autogenous bone grafting.

\section{Use of Instrumentation}

Instrumentation in the pediatric cervical spine has similar indications to that of an adult spine. Instrumentation was developed to achieve higher cervical fusion rates, reduce graft extrusion rates, decrease the need for external orthotic immobilization, and help restore and maintain proper spinal alignment. ${ }^{23}$ In all children or adolescents who may undergo an anterior cervical procedure in which spinal instrumentation might be placed, the preoperative workup should include plain radiography, CT scanning, and magnetic resonance imaging. Use of CT scans with thin cuts and three-dimensional reconstructions is now commonplace, and these neuroimaging studies are mandatory in assessing whether a child's VBs are large enough to accommodate standard instrumentation. Dynamic radiographs should be considered in nontraumatic cases or situations without obvious instability. Miniplates may need to be considered in younger or smaller children.

Theoretically, the use of plating systems may reduce the incidence of nonunion and shorten the time to achieve bone fusion. The plate may also assist the construct by resisting a variety of potentially deleterious forces, including rotational, lateral bending, and flexion-extension forces. Additionally, when the bone graft is being held in contact under the load, the plate becomes part of the load-bearing crosssectional area, adding further stability to the construct. ${ }^{23}$

Plating systems may be useful in maintaining cervical alignment and reducing the incidence of graft extrusion, and they may prevent late kyphotic deformity. A late kyphotic deformity may occur in up to $50 \%$ of patients treated with bone grafting alone in the setting of trauma and secondary cervical instability. ${ }^{31}$ Use of a plating system may, in some instances, also reduce the need for posterior stabilization procedures. Placement of anterior cervical plates may also be the optimal method of fixation for distractionextension injuries, in which the plate serves as an anterior tension band over the applied graft. This theory has been supported by a number of retrospective studies, although biomechanical and cadaveric studies consistently support the need for supplemental posterior fixation in cases of global instability.

Anterior cervical instrumentation is not without significant risks. Potential complications include the risk of injury to nerve roots, implant loosening and migration, injury to adjacent structures, junctional spondylosis, stenosis, and possible instability resulting from the rigidity of the construct. ${ }^{31}$ Additionally, these devices add considerably to the cost of surgery. Nevertheless, the cost may be offset by avoiding the potential complications of kyphosis and pseudarthrosis. Meticulous site preparation and correct plate selection are critical for successful plate placement. Any soft tissue overlying the anterior VB should be removed. Additionally, the plate should be of appropriate length so that its screw holes correspond to the region of the mid-VB. A plate should also never cross an unfused disc space, which would result in excessive motion of the plate segment, predisposing it to loosening. ${ }^{23}$ Finally, a plate should never be a substitute for biomechanically sound principles.

Vaccaro, et al. ${ }^{33}$ noted a 9\% dislodgment rate for graft plates in patients undergoing two-level corpectomy fusion, but a $50 \%$ rate of graft plate dislodgment in patients undergoing three-level corpectomy fusion. Thus, for corpectomies covering three or more levels, posterior stabilization should also be considered. Numerous other implants have been used in the adult cervical spine, including titanium mesh and PEEK cages. Although they provide advantages, including reduced donor site morbidity and immediate load-bearing support, possible drawbacks of these devices also need to be mentioned. These include increased cost, potential difficulty assessing fusion radiographically, stress shielding, and potential serious soft-tissue injury should a titanium cage dislodge. If a cage is to be used, a plate should be used to minimize the risks of dislodgment. Nevertheless, titanium mesh cages may have a role in vertebrectomy for malignancy in the older adolescent, as may expandable cages.

Polyetheretherketone cages have been used with and without BMPs in the cervical spine, with good results. ${ }^{5,9}$ Nevertheless, given the well-described inflammatory response seen in some cases of PEEK cage implantation, this may not be the best choice in the pediatric population. ${ }^{17,30}$ Additionally, the use of BMP in the pediatric cervical spine needs to be studied further before it can be recommended, especially given anecdotal reports of swelling in the neck following the use of BMP in the anterior cervical spine. ${ }^{5}$

Recently, resorbable plates have been approved for use in the cervical spine (Fig. 2). A retrospective study of nine patients undergoing ACDF with resorbable mesh plate was published, which showed good clinical and radiographic results. ${ }^{35}$ This has a potentially great benefit for the pediatric population, in that a more physiological modulus of elasticity of the instrumentation is seen in relation to native vertebral bone compared with different metallic devices. Additionally, no radiological artifacts are seen when assessing the fusion. More recently, resorbable spacers have been used with ACDF, with good results. ${ }^{34}$

Resorbable plates degrade in a predictable way, which eliminates residual metal instrumentation and the potential complications later in life of migration or plate dislodgment. Additionally, they eliminate stress shielding by their gradual 


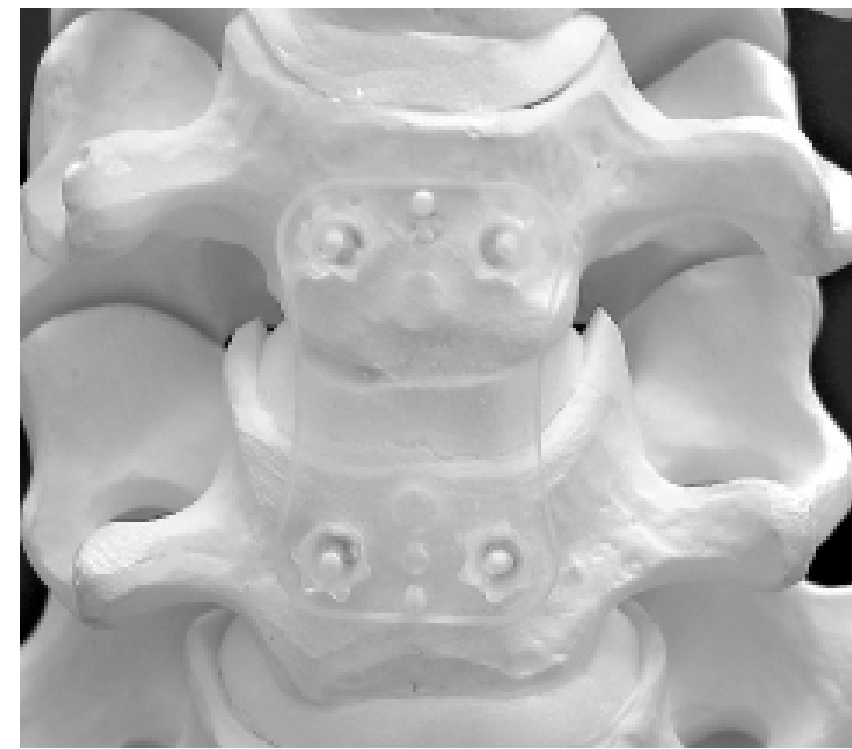

FIG. 2. Photograph of a spine model with the Mystique bioabsorbable plate (Medtronic Sofamor Danek, Memphis, TN) in place. This plate is made from a copolymer of $70 \%$ poly(L-lactide) and $30 \%$ poly(D,L-lactide), for which resorption typically occurs in a little more than 18 months.

dissolution. Dickerman, et al. ${ }^{13}$ used a sheet of Macropore (which has been demonstrated to incorporate in vivo within 18 months) for this purpose in a young child undergoing cervical corpectomy. A recent biomechanical study using cadaveric cervical spines and Macropore plates found the plate to be effective at preventing graft extrusion. ${ }^{2}$ For a single-level procedure, the plates did not confer additional stability over bone grafting alone. For two-level ACDF, however, the plates significantly increased stability. How these plates compare with titanium in terms of fusion rates and clinical outcomes remains to be seen.

Disc arthroplasty may have a role in the treatment of older adolescents with disc herniation. Although theoretically disc arthroplasty may maintain normal motion and decrease the likelihood of adjacent segment disease, longterm outcomes need to be studied further in the adult population before these procedures can be recommended for general pediatric use.

\section{Conclusions}

Due to different disease patterns from those seen in the adult population, the anterior cervical approach is rarely indicated in children. Most commonly it is used with or without instrumentation for trauma, deformity, and for tumor resection. Similar instrumentation principles apply to the pediatric population as to the adult population. A careful review of preoperative imaging must be undertaken to size the instrumentation appropriately. Bioabsorbable materials potentially have a role in the treatment of pediatric cervical spine disease. Although good long-term results have been seen, patients should be observed closely for the subsequent development of postsurgical spinal deformity, which theoretically may necessitate further intervention.

\section{References}

1. Allsopp GM, Griffiths S, Sgouros S: Cervical disc prolapse in childhood associated with Klippel-Feil syndrome. Childs Nerv Syst 17:69-70, 2001

2. Ames CP, Crawford NR, Chamberlain RH, et al: Biomechanical analysis of a resorbable anterior cervical graft containment plate. Spine 30:1031-1038, 2005

3. Bailey RW, Badgley CE: Stabilization of the cervical spine by anterior fusion. J Bone Joint Surg Am 42:565-594, 1960

4. Banks JT, Wellons JC III, Tubbs RS, et al: Cervical spine involvement in Larsen's syndrome: a case illustration. Pediatrics 111:199-201, 2003

5. Boakye M, Mummaneni PV, Garrett M, et al: Anterior cervical discectomy and fusion involving a polyetheretherketone spacer and bone morphogenetic protein. J Neurosurg Spine 2: 521-525, 2005

6. Brockmeyer D, Apfelbaum R, Tippets R, et al: Pediatric cervical spine instrumentation using screw fixation. Pediatr Neurosurg 22:147-157, 1995

7. Casey AT, Hayward RD, Harkness WF, et al: The use of autologous skull bone grafts for posterior fusion of the upper cervical spine in children. Spine 20:2217-2220, 1995

8. Chadduck WM, Boop FA: Use of full-thickness calvarial bone grafts for cervical spinal fusions in pediatric patients. Pediatr Neurosurg 20:107-112, 1994

9. Cho DY, Liau WR, Lee WY, et al: Preliminary experience using a polyetheretherketone (PEEK) cage in the treatment of cervical disc disease. Neurosurgery 51:1343-1350, 2002

10. Cloward RB: The anterior approach for removal of ruptured cervical discs. J Neurosurg 15:602-617, 1958

11. Deburge A, Briard JL: Cervical hemivertebra excision. J Bone Joint Surg Am 63:1335-1338, 1981

12. DePalma AF, Rothman RH, Lewinnek GE, et al: Anterior interbody fusion for severe cervical disc degeneration. Surg Gynecol Obstet 134:755-758, 1972

13. Dickerman RD, Morgan JT, Mittler M: Circumferential cervical spine surgery in an 18-month-old female with traumatic disruption of the odontoid and C3 vertebrae. Case report and review of techniques. Pediatr Neurosurg 41:88-92, 2005

14. Fielding JW, Pyle RN Jr, Fietti VG Jr: Anterior cervical vertebral body resection and bone-grafting for benign and malignant tumors. A survey under the auspices of the Cervical Spine Research Society. J Bone Joint Surg Am 61:251-253, 1979

15. Hoffmann RF, Weisskopf M, Stockle U, et al: Bisegmental rotational fracture dislocation of the pediatric cervical spine. A case report. Spine 24:904-907, 1999

16. Holmes JC, Hall JE: Fusion for instability and potential instability of the cervical spine in children and adolescents. Orthop Clin North Am 9:923-943, 1978

17. Jockisch KA, Brown SA, Bauer TW, et al: Biological response to chopped-carbon-fiber-reinforced peek. J Biomed Mater Res 26:133-146, 1992

18. Johnston CE II, Birch JG, Daniels JL: Cervical kyphosis in patients who have Larsen syndrome. J Bone Joint Surg Am 78: 538-545, 1996

19. Lester JW Jr, Miller WA, Carter MP, et al: MR of childhood calcified herniated cervical disk with spontaneous resorption. AJNR Am J Neuroradiol 10:S48-S50, 1989

20. Lustrin ES, Karakas SP, Ortiz AO, et al: Pediatric cervical spine:normal anatomy, variants, and trauma. Radiographics 23: 539-560, 2003

21. Malloy KM, Hilibrand AS: Autograft versus allograft in degenerative cervical disease. Clin Orthop Relat Res 394:27-38, 2002

22. McGregor JC, Butler P: Disc calcification in childhood: computed tomographic and magnetic resonance imaging appearances. Br J Radiol 59:180-182, 1986

23. Papadopoulos SM: Anterior cervical instrumentation. Clin Neurosurg 40:273-285, 1993 
24. Piccirilli CB, Chadduck WM: Cervical kyphotic myelopathy in a child with Morquio syndrome. Childs Nerv Syst 12:114-116, 1996

25. Schaser KD, Stover JF, Kaeaeb MJ, et al: Mild cervical spine trauma showing symptomatic calcified cervical disc herniation in a child: a case report. Spine 28:E93-E94, 2003

26. Shacked I, Ram Z, Hadani M: The anterior cervical approach for traumatic injuries to the cervical spine in children. Clin Orthop Relat Res 292:144-150, 1993

27. Smith GW, Robinson RA: The treatment of certain cervicalspine disorders by anterior removal of the interbody disc and fusion. J Bone Joint Surg Am 40:607-624, 1958

28. Sohn MJ, Park HC, Park HS, et al: Anterior cervical corpectomy and fusion using miniplate and screws in a 7-year-old child with eosinophilic granuloma of the cervical spine. Spine 26: 1193-1196, 2001

29. Stauffer ES, Kelly EG: Fracture-dislocations of the cervical spine. Instability and recurrent deformity following treatment by anterior interbody fusion. J Bone Joint Surg Am 59:45-48, 1977

30. Toth JM, Wang M, Estes BT, et al: Polyetheretherketone as a biomaterial for spinal applications. Biomaterials 27:324-334, 2006

31. Vaccaro AR, Balderston RA: Anterior plate instrumentation for disorders of the subaxial cervical spine. Clin Orthop Relat Res 335:112-121, 1997
32. Vaccaro AR, Cirello J: The use of allograft bone and cages in fractures of the cervical, thoracic, and lumbar spine. Clin Orthop Relat Res 394:19-26, 2002

33. Vaccaro AR, Falatyn SP, Scuderi GJ, et al: Early failure of long segment anterior cervical plate fixation. J Spinal Disord 11: 410-415, 1998

34. Vaccaro AR, Robbins MM, Madigan L, et al: Early findings in a pilot study of anterior cervical fusion in which bioabsorbable interbody spacers were used in the treatment of cervical degenerative disease. Neurosurg Focus 16:E7, 2004

35. Vaccaro AR, Venger BH, Kelleher PM, et al: Use of a bioabsorbable anterior cervical plate in the treatment of cervical degenerative and traumatic disk disruption. Orthopedics 25: S1191-S1199, 2002

36. Wickboldt J, Sorensen N: Anterior cervical fusion after traumatic dislocation of the cervical spine in childhood and adolescence. Childs Brain 4:120-128, 1978

Manuscript received November 27, 2005.

Accepted in final form January 10, 2006.

Address reprint requests to: Devanand A. Dominique, M.D., Department of Neurosurgery, Temple University Hospital, 5th Floor Parkinson Pavilion, 3401 North Broad Street, Philadelphia, Pennsylvania 19140. email: ddominiq@ temple.edu. 